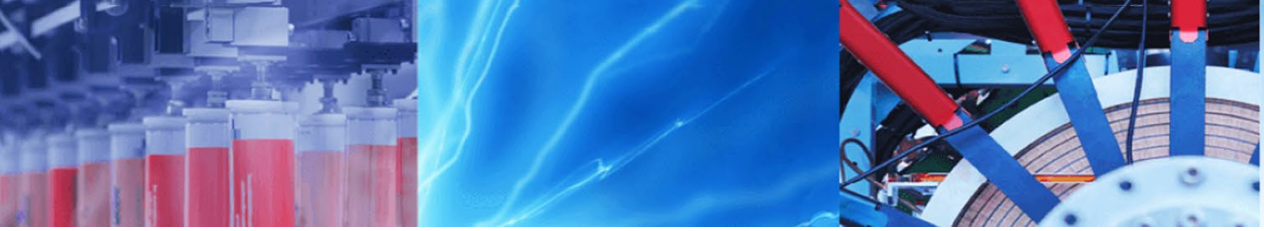

Research Article

\title{
Efficient paradigm to enhance particle separation in deterministic lateral displacement arrays
}

\author{
Amirali Ebadi ${ }^{1} \cdot$ Mohammad Javad Farshchi Heydari $^{1,2} \cdot$ Reihaneh Toutouni $^{1,3} \cdot$ Bahareh Chaichypour $^{1,2}$. \\ Morteza Fathipour ${ }^{1} \cdot$ Kian Jafari $^{4}$
}

(c) Springer Nature Switzerland AG 2019

\begin{abstract}
Microfluidics based particle sorting and separation methods are gaining momentum to be applied for various applications. Deterministic lateral displacement (DLD) methods are prominent for high resolution in separation and there has been extensive studies to develop more efficient devices based on the DLD. However, it is still challenging to fully eliminate negative effects of the boundaries that degrade particle separation efficiency by perturbing the fluid flow in the channel. In this article, we present two equations to optimize channels' geometry near the boundaries. Implementing the equations, the fluid behavior is improved around the pillars and thereby, separation efficiency is increased. The Boundary Correction Paradigm (BCP) enhances the microchannel's functionality as much as $2-3$ times and can be highly beneficial in microchannels. Also, an equation is proposed in order to recalibrate the BCP in microchannels with desired pillar diameters. The calibration equation assures high accuracy and resolution of the DLD devices corrected with the BCP.
\end{abstract}

Keywords Microfluidics · Deterministic lateral displacement · Particle separation efficiency · Boundary correction paradigm

\section{Introduction}

One of the mostly employed capabilities of microfluidics towards accomplishment of Lab-on-a-chip technology is separation of specific bio particles in a whole sample. This application paves the way for diagnosing different diseases and developing efficient therapeutics. Separation methods and particle analysis are two significant steps need to be swift, low-cost, efficient, and continuous [1-6]. Microfluidics is widely utilized for this purpose and enables separation of target particles with low concentration in biofluids like blood [7]. The separation of bioparticles on microfluidic platforms mostly relies on particle physical properties like mass, size, charge, etc. and mainly fall into two categories of active and passive [8-10]. Active sorting is implemented on devices that use external fields to manipulate and act on the particles and the flow. Some of the active separation methods include dielectrophoresis, magnetophoresis, and acoustophoresis. Dielectrophoresis [11-13] sorts particles based on their size and capacitance by imposing variant electric field in the channel. Acoustophoresis transmits bulk $[14,15]$ or surface [16] ultrasound waves so that the particles in the channel would be separated based on differences in size, density, and compressibility [17]. Magnetophoresis offers particle sorting based on the magnetic susceptibility of the particles which could be intrinsic [18] or superparamagnetic $[19,20]$. On the contrary, passive methods mainly rely on channel geometry and hydrodynamic forces for separation which their simplicity and throughput is comparable to active methods [21]. Passive methods include, but are

$\triangle$ Morteza Fathipour, mfathi@ut.ac.ir| ${ }^{1}$ Department of Electrical and Computer Engineering, University of Tehran, Tehran 1439957131, Iran. ${ }^{2}$ Department of Mechanical Engineering, K. N. Toosi University of Technology, Tehran 1991943344, Iran. ${ }^{3}$ Department of Petroleum Engineering, Wyoming University, Laramie 82071, USA. ${ }^{4}$ Electrical Engineering Department, Shahid Beheshti University, (SBU),

Tehran 1983969411, Iran. 
not limited to, hydrodynamic or hydrophoretic filtration, pinched-flow fractionation (PFF), and deteministic lateral displacement (DLD) [8, 9, 22-24]. The DLD, first proposed by Huang et al. [25], sorts particles based on their diameters. This method is used for variety of purposes such as separating blood components [26-29], measuring platelet activity [30], separating circulating tumor cells (CTCs) [31-33], spores [34], bacteria [35], parasites [36] and even nanoparticles [37, 38]. Compared to other methods, the DLD is cost-efficient, easy to use, accurate, and leaves morphology and properties of the particles intact. In addition, it has highest resolution among separation methods [25].

In the DLD method, the channel's geometry is of high importance. The design and arrangement of pillar arrays play the main role in separation. The angle of pillar arrays relative to the flow direction is called migration angle $(\theta)$. Also, the gap $(\mathrm{G})$ between each two pillars are equal. The gap $(G)$ and migration angle $(\theta)$ define a critical size $\left(D_{c}\right)$ for separation. Therefore, particles will follow either zigzag or bumping streamlines $[25,39]$. Particles with smaller diameters than the $D_{c}$ will go through zigzag mode with approximately no lateral displacement. On the contrary, particles with larger diameters than the $D_{c}$ will have bumping trajectory and migrate upward $[25,39]$. In this way, the particles with diameters less and over the critical diameter $\left(D_{c}\right)$ will be separated.

One of the challenges in the DLD method, however, is particle clogging near the boundary interfaces. According to the DLD theory [25], it is assumed that there are no boundaries to disturb the Poiseuille flow and particle separation. Due to limitations in the fabrication and inevitable presence of boundaries, the Poiseuille flow pattern is disturbed in experimental setups. Therefore, anticipated streamlines and critical diameters $\left(D_{c_{\mathrm{t}}}\right)$, according to the DLD theory, won't be realized in the experiment. Each pillar in the array would have a different $D_{c}$ and the pillars close to the boundaries would malfunction, thereby, zigzag and bump streamlines will be merged and particles of all sizes flow near walls.

Inglis et al. [40] proposed a method to resolve the problem. They modified the distance between the boundaries and the pillars close to them. In this way, the flow pattern in the channel improved and numerical simulation showed that resulted critical diameters came closer to $\left(D_{c_{t h}}\right)$. The method included two equations for modifying the two boundaries. The Eqs. 1 and 2 presented by Inglis et al. to correct boundary interfaces are expressed for left and right boundaries respectively [40] for a channel with downward flow,

$g_{\mathrm{n}}=G \sqrt{n \varepsilon}$

$g_{\mathrm{n}}=G \sqrt{2-n \varepsilon}$ where $g_{n}$ is the distance of each boundary-adjunct pillar to its neighboring wall, $\mathrm{G}$ is the distance between two pillars in a row, $\varepsilon$ is pillars row incline and $\mathrm{n}$ can be a number from 1 to $1 / \varepsilon$. Employing the equations to implement wall correction has led to a better performance of the DLD devices. Flow patterns enhanced to be more similar to the Poiseuille. In another attempt to improve DLD-based separation efficiency, Feng et al. [41] proposed a design in which used two DLD arrays mirrored so that smaller particles would focus in the middle and bigger particles would bump toward boundaries. They also indicated that the flow flux passing through a DLD channel is proportional to the cube of the gap sizes in the channel, comparable to the square proportion that was previously expressed [40].

In this paper a more efficient wall correction method based on the original theory is proposed to further bridge the gap between theory and practice. Similar to the Inglis et al.'s method, the Boundary Correction Paradigm (BCP) also includes two equations for the microchannel boundary correction. Derivation of the BCP's equations are first explained. Afterward, on a completely similar microchannel, the BCP and Inglis et al.'s method are used to correct the boundaries and then the fluid streamlines and resulted critical diameters by both methods are compared. The BCP showed to enhance homogeneity of streamlines and critical diameters in the microchannel. Also, the critical diameters converged to the $D_{c_{\mathrm{th}}}$.

\section{Materials and methods}

\subsection{Theory of DLD}

The DLD was first described by Hunag et al. [25], using an array of pillars arranged to have an offset relative to the flow direction in microchannel (See Fig. 1. Size-based sorting of the particles in the carrying fluid can be carried out while passing the pillars. A critical size for the separation, critical diameter $\left(D_{c}\right)$, is determined by two design parameters of the gap size between the pillars and the angle between the pillar array and the flow direction. Particles with smaller diameters than $D_{c}$ zigzag through the pillars with almost no movement along $y$-direction whereas those with bigger diameters bump on the pillars and would have a lateral displacement. Consequently, particles are distinguished based on their size and the sorting is accomplished. There are several parameters that should be taken into consideration in the DLD array design. As mentioned, the arrays have an offset relative to the flow direction which is defined as inclination angle $\left(\theta=\tan ^{-1} \varepsilon\right)$ where $\varepsilon$ is the slope. $\lambda$ is the center to center distance of neighboring pillars in a column and the shift between two pillars in consecutive columns is $\lambda \varepsilon$. The number of pillars 


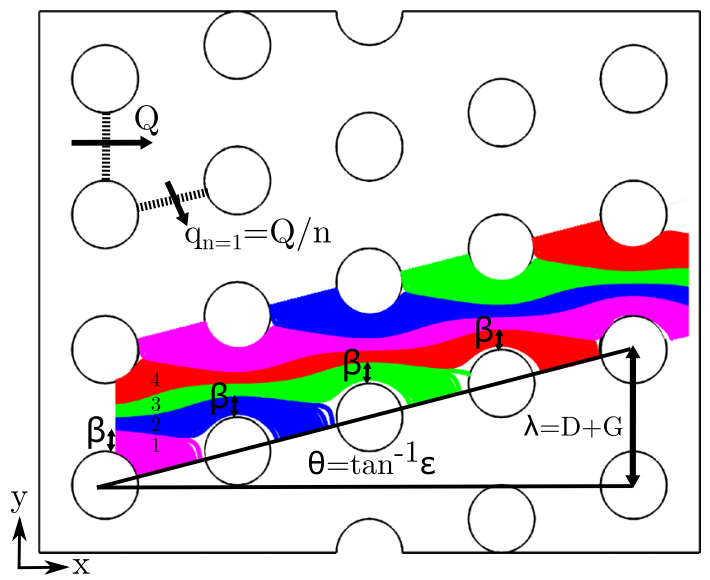

Fig. 1 Schematic array of a DLD device consisting $n=4$ columns to depict the original theory and geometry characteristics. $\beta$ is shown which is first flow width and equals $D_{c} / 2$. $\lambda$ equals sum of diameter and gap sizes. $\mathrm{Q}$ is the flux passing between two vertically neighboring pillars and $\mathrm{q}_{\mathrm{n}}$ passes two horizontally neighboring pillars

in a row can be expressed as an integer $(n=1 / \varepsilon)$. Furthermore, the flow flux between each two pillars in a column can be divided to $n$ parts (See Fig. 1, an array with $n=4$ is considered) and the first flow stream width would be $\beta$. Finally, the critical diameter is defined as twice the $\beta$ $\left(D_{c}=2 \beta\right)$. First flow stream can be expressed as integration of flow profile $u(x)$, where $g$ is the gap,

$\int_{0}^{\beta} u(x) d x=\varepsilon \int_{0}^{g} u(x) d x$

Also, in creeping flow conditions with no-slip in the microchannel, flow profile can be expressed as

$u(x)=\left[\frac{g^{2}}{4}-\left(x-\frac{g}{2}\right)^{2}\right]$.

Using 4 in 3 , we would have $\beta$ to calculate $D_{c^{\prime}}$

$\left[\frac{\beta}{g}\right]^{3}-\frac{3}{2}\left[\frac{\beta}{g}\right]^{2}+\varepsilon \frac{1}{2}=0$.

Using 5, the critical diameter for an ideal DLD device can be achieved $\left(D_{c_{\mathrm{th}}}\right)$ [39]. The aforementioned characteristics are depicted in a DLD device with $n=4$ in Fig. 1. It can be established that the flow is divided to $n(=1$ to 4$)$ parts that each pass beneath $n^{\text {th }}$ column. For instance, first stream (pink) flows beneath first column; second stream (blue) flows below the second column and so on. Also, it can be established that this pattern applies for upper rows. It is noteworthy to mention that the aforementioned equations were presented by the pioneers of the DLD field which was a grand breakthrough. However, there is a large gap between the theory and experimental setups for several reasons. First, the DLD theory explains an ideal flow within infinite parallel plates which are impossible to be realized in experiments. Second, the DLD theory describes the flow profile only in two dimensions and the third dimension is neglected which brings about disharmony from the original theory even in large devices. The non-ideal conditions like finite boundary interfaces in experimental devices make the calculated $D_{c}$ far to achieve in experiment [42].

\subsection{Improving effect of using obround pillars before the DLD array}

The obround pillars are mainly used after the inlet and before the pillar arrays in several past works [41] but the explanation of their positive effect was ignored. Here, we are going to discuss and numerically explain how using these pillars can improve functionality of every DLD device. As the pillars are similar throughout the channel, the pressure gradient before and after each pillar along the $y$-direction should be constant. However, this condition is not fulfilled across the initial pillars after the inlet. As a result, the efficiency is plummeted in the initial section. As an initial attempt to improve the DLD function, we have studied the influence of obround pillars on making the pressures uniform after the fluid inlet by the Finite Element Method (FEM). To elaborate, before the pillar arrays, the flow is Poiseuille-like, fully developed and has maximum velocity in the middle of the channel. It runs into the micro pillars and as a result, the flow velocity (thus pressure drop) is higher in the middle of the channel through the first columns of pillars (See Fig. 5b). Thereby, the critical diameters are only homogeneous after the few first columns and those columns would be futile. To overcome this velocity non-uniformity through the first columns, obround pillars are located before the DLD array exactly on the same $y$-coordinates as the first column of pillars (See Fig. 5b). Consequently, the flow is stabilized before it enters the pillars network and the first columns would be beneficial as well. Figure 2 displays pressure profiles in the channel's width in two conditions of with and without obround pillars in a sample DLD array having 17 rows. Moreover, after studying pressure values along the $y$-axis between every two horizontally neighboring pillars in Fig. 2c, it is deduced that the pressure has become constant and has no fluctuations when the obround pillars are used.

\subsection{Derivation of boundary correction equations}

It is learned from the previous section that pressure difference $(\Delta P)$ between consecutive pillars in a row is constant as depicted in Fig. $2 c$ (pressure line is flat along the $y$-axis). 

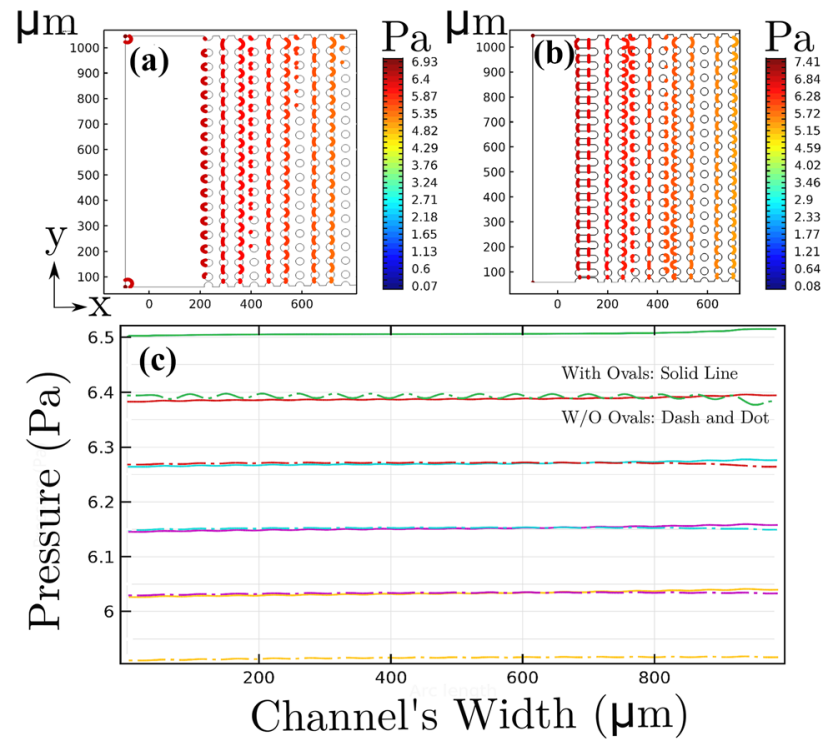

Fig. 2 Depiction of pressure profiles $\mathbf{a}$ with and $\mathbf{b}$ without obround pillars present ahead of separation arrays. It is deduced that the pressure drop is increased by 6.4 percent when obround pillars are implemented. c Investigating pressure values in channel's width (along $y$-axis), it is deduced that the pressure is constant and has no oscillation when obround pillars are used. For instance, the green solid and dashed lines illustrate pressure values after the first column in microchannels with and without obround pillars, respectively. Likewise, red, light blue, purple, and yellow lines represent the measured pressure values after second, third, forth, and fifth columns, respectively, along channels' width

Thereby, we assumed that all pillars in a column (along $y$-axis) have similar $\Delta P$ values around them so we can consider only two micro pillars and simulate the flow between them assuming constant pressure on vertical boundary of both sides (See Fig. 3a). In the Poiseuille governed flows, the flux alters linearly when the magnitude of $\Delta P$ varies, which is known as Hagen-Poiseuille law [43]. To yield an optimized equation for wall correction, the optimal distance of the boundary and its adjunct micro pillar should be calculated which is also referred to as modified gap size in this manuscript. For this purpose, the two semi circles representing half of two micropillars are considered. Our ultimate goal on this numerical analysis is to discover the relation between flow flux passing through the pillars and modified gap sizes. In this simulation the pillar diameter is considered constant and equal to 40 micrometers. The gap size (G) inside the DLD array is constant and equal to 40 micrometers. The modified gap size between two pillars $\left(g_{n}\right)$ is increased from 0 to 40 with the intervals of 0.5 micrometers. The relative flux $(\mathrm{Q})$ for each quantity of gap size is then calculated. This simulation's output is employed to derive the relation of flux and modified gap sizes. In the following, developing process of wall correction method for both upper and lower wall is elaborated. As the DLD theory is based on the creeping flow, inertial term is neglected in our numerical studies.

Although it is not practical to realize unlimited boundaries for the channel, the wall correction methods attempt to accomplish this boundary condition as much as possible. To obtain an efficient wall correction method, it is required to comprehend flow behavior, especially near channel walls and boundaries. Therefore, two different equations must be developed for the lower and upper walls. These equations are developed by curve fitting of flow flux profile data passing through modified gap quantities, calculated in the last section. Among various curve fitting possibilities, the least error was obtained by power equation with two variables. Having these assumptions and the following comprehensions, the equations are developed.

\subsubsection{Lower wall equation}

Considering the lower wall and its nearing pillar row, it is established that the downward flow passing the first gap is $Q / n$, the second gap is $2 Q / n$ till the $n$th gap, that equals $\mathrm{G}$, will be $\mathrm{nQ} / \mathrm{n}$. Therefore, for the first gap we have 6 ,

$g_{1}=a q_{1}^{\frac{1}{b}}$

where $g_{1}$ is the first gap, $q_{1}$ is the flux passing below the first gap and "a" and " $b$ " are the two variables for the power equation. Thus, according to 6 , we have 7 for $G$ respectively,

$G=a Q^{\frac{1}{b}} \rightarrow Q=\left(\frac{G}{a}\right)^{b}$

Moreover, as mentioned, the flux passing below the first gap is as 8 ,

$q_{1}=\frac{Q}{n}=\varepsilon Q$

Also, it is aforementioned that $\frac{1}{n}=\varepsilon$. Utilizing $q_{1}$ from 8 and Q form 7 in 6,

$g_{1}=a\left(\frac{Q}{n}\right)^{\frac{1}{b}}=a\left(\frac{1}{n} \times\left(\frac{G}{a}\right)^{b}\right)^{\frac{1}{b}}=G \frac{1}{n^{\frac{1}{b}}}=G \varepsilon^{\frac{1}{b}}$

In the next gap (second gap), the flux is increased by $\mathrm{Q} / \mathrm{n}$. The same amount of flow is added through the next gaps until the last gap. For the general condition, from 9 we would have 10 for the $n_{t h}$ modified gap in the lower boundary,

$g_{n}=G(n \varepsilon)^{\frac{1}{b}}=\sqrt[b]{G(n \varepsilon)}$

Consequently, it was clarified that the "a" variable in 6 will not have any effect on the final wall correction equation 
(a)

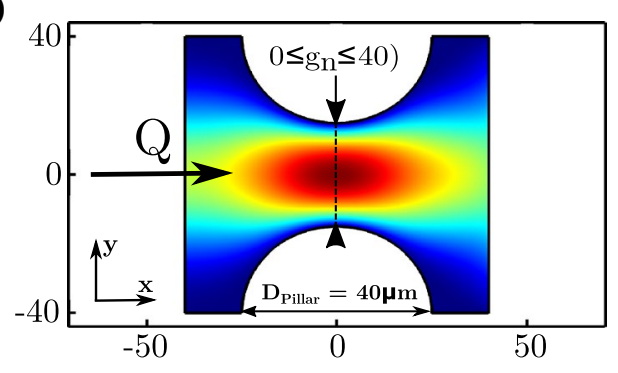

(c)

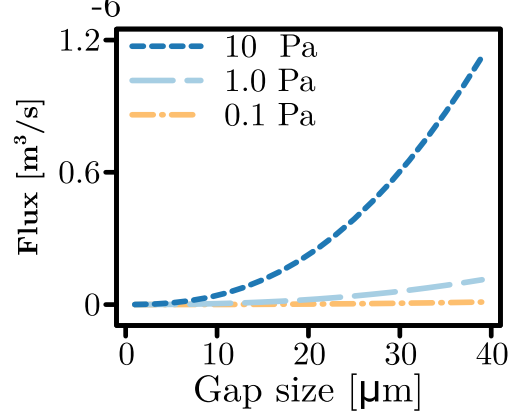

(b) -8

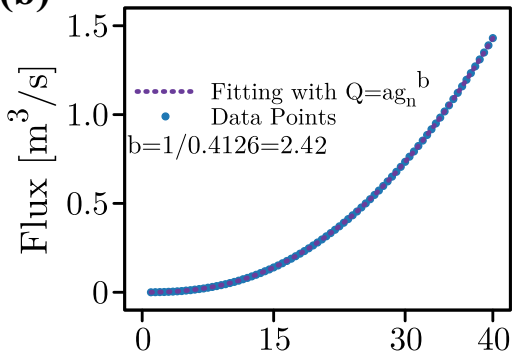

(d)

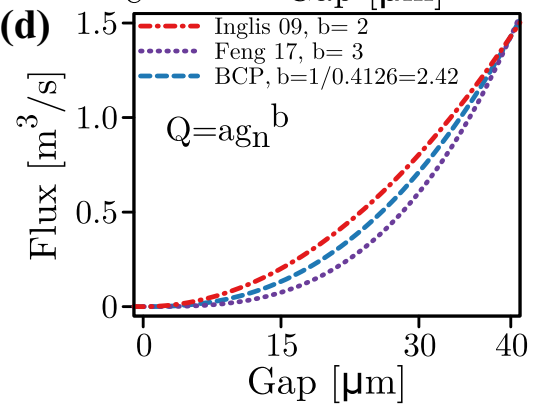

Fig. 3 a Calculation of flux passing through the modified gap sizes $\left(g_{n}\right)$ varying from 0 to $40(=G) \mu \mathrm{m}$ between two semi-pillars to achieve optimized wall correction paradigm. The fluid is assumed to flow from left to the right. $\mathbf{b}$ The data corresponding obtained flux values for each gap size is plotted by blue dots. To obtain the $\mathrm{BCP}$ paradigm, power equation $\left(Q=a g_{n}^{b}\right)$ is utilized in curve fitting to find the relation of modified gap sizes and the flux passing through them. As a result of power fitting the " $b$ " variable is computed to be 2.463 (or 1/0.4126). c The calculation is repeated when pressure difference $(\Delta P)$ between left and right edges are $0.1,1$ and $10 \mathrm{~Pa}$. By the figure, it is established that the flux values for each modified gap are timed to 10 and 100 as the $(\Delta P)$ is timed to 10 and 100 , respectively. Thereby, when power equation

and only one variable " $b$ " plays the only role and should be determined.

\subsubsection{Upper wall equation}

For upper wall, as the large particles flow toward above the channel, larger gaps are considered to prevent clogging. Therefore, passing flow from the first gap is $Q+Q / n$, from the second gap is $Q+2 Q / n$, and goes on till the $n$ thgap, equaling $\mathrm{G}$, that has $2 \mathrm{Q}$ passing flow. Therefore, similar to lower wall equations, we have,

$g_{1}=a q_{1}^{\frac{1}{b}}$

$G=a Q^{\frac{1}{b}} \rightarrow Q=\left(\frac{G}{a}\right)^{b}$

We also know that,

$q_{1}=2 Q-\frac{Q}{n}=(2-\varepsilon) Q$ $\left(y=a x^{b}\right)$ is employed for curve fitting, the "a" variable is affected (as a result of implemented changes in $\Delta P$ ) and " $b$ " variable is left intact. d By this figure, a comparison between the presenting $B C P$ paradigm and previous methods in theory is carried out. For the equation of $Q=a g_{n^{\prime}}^{b}$ Inglis et al. [40] proposed $b=2$ (red), Feng et al. [41] proposed cubic root to explain the relation (purple), and hereby we propose $b=2.463$ in the BCP paradigm (blue). Assuming $Q=1 \mathrm{~m}^{3} / \mathrm{s}$ and acknowledging $g_{0}=0$ and $g_{n=40}=40$, we obtained "a" for each method and plotted each equation to exhibit the difference. It is established that the BCP lies almost between previous two methods to describe the relation of modified gap sizes and the flux passing through them

First employing $q_{1}$ from 13 in 11 and G from 12 in 11,

$g_{1}=a\left(\frac{Q}{n}\right)^{\frac{1}{b}}=a((2-\varepsilon) Q)^{\frac{1}{b}}=a\left((2-\varepsilon)\left(\frac{G}{a}\right)^{b}\right)^{\frac{1}{b}}=G(2-\varepsilon)^{\frac{1}{b}}$

And for the general condition, gap size for $\mathrm{n}^{\text {th }}$ column in upper wall is developed in 15.

$g_{n}=G(2-n \varepsilon)^{\frac{1}{b}}=\sqrt[b]{G(2-n \varepsilon)}$

Again, it was determined that the "a" variable in 11 is omitted in final equation for upper wall (15); and variable "b" should be found to hand in the equation.

\section{Results and discussion}

In this section, derivation of the BCP and it's two equations and variables are elaborated. Also, a microchannel's boundaries are corrected by BCP and Inglis et al.'s method 
and resulting flow patterns and critical diameters $\left(D_{c}\right)$ are compared.

\subsection{Determination of BCP equations' variables}

As the equations are developed, derivation of variables to find the optimized wall correction method are essential. To do so, the obtained data from simulation of modified gaps and their relative flux are used. The curve fitting of the results with power equation pattern $\left(y=a x^{b}\right)$ with two variables resulted in the least error. However, this question was raised that how changing basic boundary conditions like $\Delta P$ affects the output. Therefore, three different $\Delta P$ values $(0.1,1,10 \mathrm{~Pa})$ were utilized to repeat the simulation and compare the outcome. Aftermath, it was observed that only quantity of "a" is influenced in power fitting by different amounts of pressure which does not affect the equation. Figure $3 \mathrm{~b}$ illustrates how flux is increased by higher pressure differences $(\Delta P)$.

Furthermore, to acquire the accurate power variable, " $b$ ", several measures are taken to ensure the reliability of output results. First, the results independency from the utilized mesh were accomplished by gradually decreasing mesh size till the results were unchanged from some point. Second, the number of modified gap sizes and the relative flux passing through them were increased to reach the highest accuracy in the curve fitting. This process was also carried on until no alteration was observed in the achieved equation. Subsequently, the " $b$ " variable was determined to be 2.463 by the curve fitting, Fig. 3c. Goodness of fit was ensured by R-square and adjusted R-square equal to 1 . We herewith propose Boundary Correction Paradigm (BCP) to improve homogeneity of $D_{c}$ in the channel. Equations 16 and 17 are presented as the BCP method to correct two boundaries.

$g_{n}=G(n \varepsilon)^{0.4126}=\sqrt[2.463]{G(n \varepsilon)}$

$g_{n}=G(2-n \varepsilon)^{0.4126}=\sqrt[2.463]{G(2-n \varepsilon)}$

The resulting equations are similar to wall correction method presented in previous research works $[40,41]$. Inglis et al. (Feng et al.) assumed that the flux passing through the modified gap sizes is proportional to square (cube) of gap size. However, hereby, the authors exhibited that a precise equation describing the relation of flux through modified gap sizes with a constant pillar size can be presented in 16 and 17 for the lower and upper boundaries, respectively.

\subsection{Calibration formula for the BCP method}

In the previous sections, we have developed two main equations for the correction of boundaries in a DLD device to maximize the separation resolution. We assumed that the pillar diameter $\left(D_{p}\right)$ is 40 micrometers in the DLD array and then calculated the flux passing through the modified gap sizes from 1 to 40 micrometers to obtain the ' $b$ ' variable in $Q=a g^{b}$ and thereby the boundary correction equations. However, the assumption of constant $D_{p}$ could bring about incompetence to the proposed $B C P$ when employed for microchannels with $D_{p}$ sizes rather than 40 micrometers. Therefore, in this section, we seek to propose another equation as a part of the BCP that calibrates boundary correction equations for microchannels with different $D_{p}$ sizes. In this way, one could calculate the ' $b$ ' variable with respect to the pillar diameters between 0 to 40 micrometers. In order to derive such equation, we have repeated to calculate the flux passing through the modified gap sizes with varying pillar diameters. For each value of $D_{p}$, the obtained flux and modified gap size are used for curve fitting to find the ' $b$ ' variable in the $Q=a g^{b}$ equation. The calculated ' $\mathrm{b}$ ' variable for each $D_{p}$ are depicted in the Fig. 4. As there are two horizontal and vertical asymptotes, we have used fractional equation in curve fitting to derive the calibration equation, expressed below.

$b=\frac{2.494 \times d+3.298}{d+1.81}$

where $d$ is the pillar diameter and $b$ is the ' $b$ ' variable used in the BCP's boundary correction equations. It is noteworthy to mention that as the ' $b$ ' in 18 approaches zero (there are no pillars in the channel), $b=\frac{3.298}{1.81} \approx 2$ which is the Poiseuille. Also, as the ' $b$ ' in 18 approaches the infinity, $b=2.494$ which is approximately equal to the proposed value for ' $b$ ' in the BCP's boundary correction equations. In brief, we proposed a calibration equation in this section so that the ' $b$ ' variable could be recalculated for microchannels with different pillar diameters. Then, the calculated ' $b$ ' variable could be used in the BCP boundary correction equations.

\subsection{Numerical analysis}

Hereby, we seek to validate the BCP approach by numerical analysis. For this purpose, finite element method (FEM) is employed. To ensure the maximum simulation accuracy, the results are validated with Inglis et al. previous work [42]. A DLD micro-channel is assumed with a pillar array of 20 columns and 8 rows which makes it a narrow device. Then, we put three identical DLD arrays (with assumed characteristics) in series along the $x$-axis to further evaluate the BCP paradigm prformance in consecutive DLD arrays. We hereby use the terms of first, second or third array to refer to the array we long to discuss in this sequence. The micro-channel's boundaries are first modified using the Inglis et al.'s method [40] principles and then with the BCP 

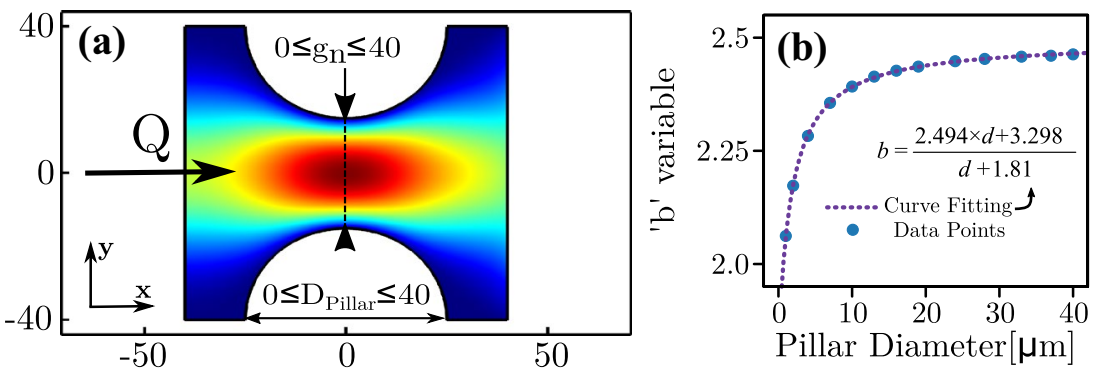

Fig. 4 a Calculation of flux passing through the modified gap sizes $\left(g_{n}\right)$ varying from 0 to $40(=G) \mu \mathrm{m}$ between two semi-pillars with varying diameters from 0 to $40 \mu \mathrm{m}$. This calculation is carried out to achieve a calibration equation in which the ' $b$ ' variable, used in the $Q=a g^{b}$, can be found for different pillar diameters. The fluid is assumed to flow from left to the right. $\mathbf{b}$ The data corresponding obtained ' $b$ ' variables for each pillar diameter size is plotted by blue dots. To obtain the calibration equation, fractional equation is utilized in curve fitting to find the relation of pillar diameter sizes and the ' $b$ ' variable. As a result of curve fitting the equation is computed to be $b=\frac{2.494 \times d+3.298}{d+1.81}$

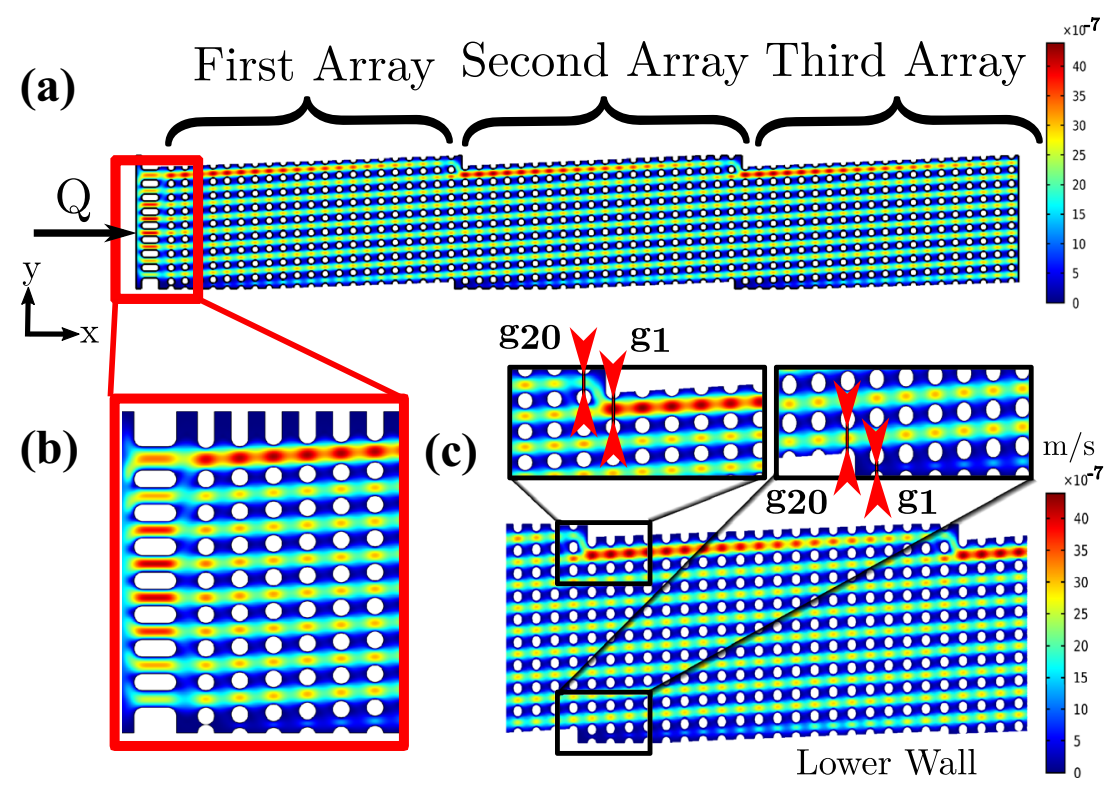

Fig. 5 a Depiction of the flux passing through three consecutive DLD arrays along the $x$-axis, simulated using finite element method (FEM). b Implementing obround pillars at the beginning of the microchannel illustrates their positive effect in stabilizing the flow pressure and velocity before entering the first array and allows taking advantage of the first few columns which would malfunc- tion without obround pillars. c The first $\left(g_{1}\right)$ and last $\left(g_{20}\right)$ modified gaps of lower and upper walls in the second DLD array are shown. The modified gap sizes in the DLD array with corrected walls using Boundary Correction Paradigm (BCP) and previous methods are studied with similar arrays

largest gap occurs in the first gap $\left(g_{n=1}=52.69 \mu \mathrm{m}\right)$ as it has to collect the fluid of two rows. And in the last column of each series, in the upper wall, $\left(g_{n=20}=40 \mu \mathrm{m}=\mathrm{G}\right)$. Figure 5 depicts the characteristics of the DLD arrays with corrected edges in Fig. 5.

As it was previously discussed by the Inglis et al. [40], the performance of the whole device will be improved when the boundaries are corrected. In order to verify the BCP method's practicality, we studied flow profile, $\mathrm{u}(\mathrm{x})$, around every micro pillar inside the three arrays which could be used interchangeably instead of the critical according to the Eq. 16 . Whereas, in the upper wall, the 
(a)

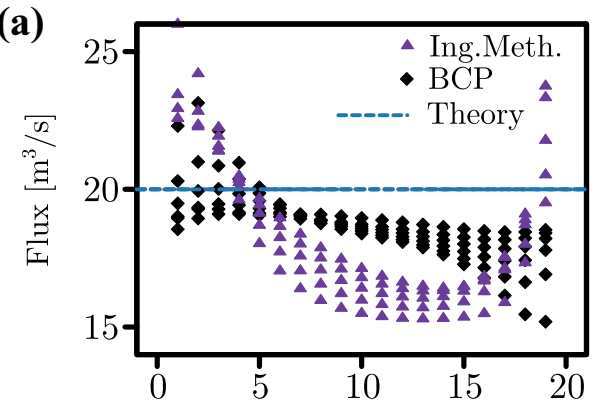

(b)

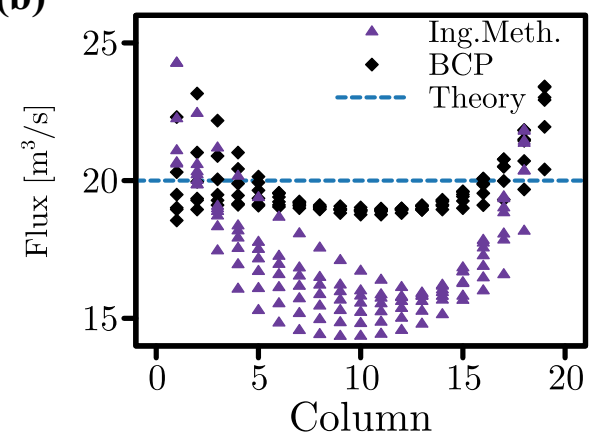

Fig. 6 Comparison of the wall correction method presented by Inglis et al. [40] (Ing. Meth.) and the Boundary Correction Paradigm $(B C P)$ in the assumed DLD device consisting three consecutive (along $x$-axis) arrays of 20 column and 8 rows which is a narrow device in a the second and $b$ the third arrays

diameter $\left(D_{c}\right)$. Using the flow profile results in reduced load of numerical analysis while does not decrease the accuracy of evaluation. Afterwards, the calculation of $Q$ for vertically neighboring pillars and $\mathrm{q}_{1}$ for horizontally adjunct pillars for both methods are carried out and the obtained values are divided $\left(\mathrm{Q} / \mathrm{q}_{1}\right)$ (See Fig. 1). This amount is expected to be 20 for a DLD array of 20 columns and is depicted by a blue line in Fig. 6 . These calculated magnitudes are illustrated in Fig. 6a, b. for the second and third arrays of the DLD device, respectively. It shows 7 quantities for each row in each column. At last, the standard deviation of this relation from the original theory is calculated for both methods by a simple equation as $\sqrt{\frac{\sum_{i=1}^{N}\left(x_{i}-x_{t h}\right)^{2}}{N}}$. where $x_{\mathrm{i}}$ is the calculated $\mathrm{Q} / \mathrm{q}_{1}, \mathrm{x}_{\mathrm{th}}$ and $\mathrm{N}$ for the assumed device are 20 and 7 respectively. It is established that the standard deviation (from the theory) was reduced from 4.15 to 2.84 in the first array, 3.51 to 2.20 in the second array, and 3.64 to 1.60 in the third array. Therefore, when the BCP method is used, closer-to-theory fluxes (critical diameters) are achieved around the pillars throughout the whole channel which is comparable to the previous methods (Fig. 6a, b). It is also deduced that the areas with the most divergence from the theory (like $g_{20}$ ) are improved in the third array compared to the second array. It is, thereby, anticipated that using even more arrays in series would result in more uniform flow in the most troubled areas. In the same manner, we exhibited how obround pillars improve the functionality of first few columns of the first array.

\section{Conclusion}

Particle separation is a significant step toward personalized medicine and lab-on-a-chip technology. Among particle separation methods, the DLD has the highest resolution and throughput compared to other methods. However, the ideal DLD boundary conditions (no boundaries to disturb the flow) cannot be implemented in the fabrication and experiment. There has been attempts to further extend the original theory and maximizing the resolution and efficiency of DLD devices by boundary correction methods. Using wall correction methods becomes crucial when the DLD arrays are utilized in nanochannels and for sensitive applications. In this paper, the Boundary Correction Paradigm (BCP) is presented to eliminate disharmonies caused by boundary interfaces which can severely affect the process of particle or cell separation when the DLD method is employed. The presented method introduces two equations to correct the boundaries and further extends and optimizes the original theory. Also, a calibration equation is proposed to assure high accuracy and resolution of the BCP in microchannels with desired pillar diameters. The standard deviation of the flow regime from the theory was enhanced $31-56 \%$ in the considered microchannel compared to previous methods.

\section{Compliance with ethical standards}

Conflict of interest On behalf of all authors, the corresponding author states that there is no conflict of interest.

\section{References}

1. Yager P, Edwards T, Fu E, Helton K, Nelson K, Tam MR, Weigl BH (2006) Microfluidic diagnostic technologies for global public health. Nature 442(7101):412

2. MacDonald MP, Spalding GC, Dholakia K (2003) Microfluidic sorting in an optical lattice. Nature 426(6965):421

3. Janasek D, Franzke J, Manz A (2006) Scaling and the design of miniaturized chemical-analysis systems. Nature 442(7101):374

4. El-Ali J, Sorger PK, Jensen KF (2006) Cells on chips. Nature 442(7101):403

5. Tabatabaei S-M, Pourfath M, Fathipour M (2018) Adsorption characteristics of epigenetically modified dna nucleobases on single-layer mos2: a first-principles study. Journal of Applied Physics 124(13):134501 
6. Tabatabaei S-M, Farshchi-Heydari M-J, Asad M, Fathipour M (2019) Unravelling the physisorption characteristics of $\mathrm{h} 2 \mathrm{~s}$ molecule on biaxially strained single-layer mos2. Nanoscale Adv

7. Smith JP, Barbati AC, Santana SM, Gleghorn JP, Kirby BJ (2012) Microfluidic transport in microdevices for rare cell capture. Electrophoresis 33(21):3133-3142

8. Autebert J, Coudert B, Bidard F-C, Pierga J-Y, Descroix S, Malaquin L, Viovy J-L (2012) Microfluidic: an innovative tool for efficient cell sorting. Methods 57(3):297-307

9. Bhagat AAS, Bow H, Hou HW, Tan SJ, Han J, Lim CT (2010) Microfluidics for cell separation. Medical Bological Engineering Computing 48(10):999-1014

10. Kumar V, Rezai P (2017) Magneto-hydrodynamic fractionation (mhf) for continuous and sheathless sorting of high-concentration paramagnetic microparticles. Biomedical Microdevices 19(2):39

11. Vykoukal J, Vykoukal DM, Freyberg S, Alt EU, Gascoyne PRC (2008) Enrichment of putative stem cells from adipose tissue using dielectrophoretic field-flow fractionation. Lab on a Chip 8(8):1386-1393

12. Song H, Rosano JM, Wang Y, Garson CJ, Prabhakarpandian B, Pant K, Klarmann GJ, Perantoni A, Alvarez LM, Lai E (2015) Continuousflow sorting of stem cells and differentiation products based on dielectrophoresis. Lab on a Chip 15(5):1320-1328

13. Adams Tayloria NG, Jiang Alan YL, Vyas Prema D, Flanagan LA (2018) Separation of neural stem cells by whole cell membrane capacitance using dielectrophoresis. Methods 133:91-103

14. Dykes J, Lenshof A, Åstrand-Grundström B, Laurell T, Scheding S (2011) Efficient removal of platelets from peripheral blood progenitor cell products using a novel micro-chip based acoustophoretic platform. PloS one 6(8):e23074

15. Lenshof A, Jamal A, Dykes J, Urbansky A, Åstrand-Grundström I, Laurell T, Scheding S (2014) Efficient purification of cd4+ lymphocytes from peripheral blood progenitor cell products using affinity bead acoustophoresis. Cytom Part A 85(11):933-941

16. Nawaz AA, Chen Y, Nama N, Nissly RH, Ren L, Ozcelik A, Wang L, McCoy JP, Levine SJ, Huang TJ (2015) Acoustofluidic fluorescence activated cell sorter. Anal Chem 87(24):12051-12058

17. Faridi MA, Ramachandraiah H, Iranmanesh I, Grishenkov D, Wiklund M, Russom A (2017) Microbubble activated acoustic cell sorting. Biomed Microdevices 19(2):23

18. Huang R, Barber TA, Schmidt MA, Tompkins RG, Toner M, Bianchi DW, Kapur R, Flejter WL (2008) A microfluidics approach for the isolation of nucleated red blood cells (nrbcs) from the peripheral blood of pregnant women. Prenat Diagn 28(10):892-899

19. Jing $Y$, Moore LR, Schneider T, Williams PS, Chalmers JJ, Farag SS, Bolwell B, Zborowski M (2007) Negative selection of hematopoietic progenitor cells by continuous magnetophoresis. Exp Hematol 35(4):662-672

20. Karabacak NM, Spuhler PS, Fachin F, Lim EJ, Pai V, Ozkumur E, Martel JM, Kojic N, Smith K, Chen P et al (2014) Microfluidic, markerfree isolation of circulating tumor cells from blood samples. Nat Protoc 9(3):694

21. Xavier M, Holm SH, Beech JP, Spencer D, Tegenfeldt JO, Oreffo ROC, Morgan $\mathrm{H}$ (2019) Label-free enrichment of primary human skeletal progenitor cells using deterministic lateral displacement. Lab on a Chip 19(3):513-523

22. Kuo J-N, Lin B-Y (2018) Microfluidic blood-plasma separation chip using channel size filtration effect. Microsyst Technol 24(4):2063-2070

23. Ebadi A, Toutouni R, Heydari Mohammad JF, Fathipour M, Soltani $M(2019)$ A novel numerical modeling paradigm for bio particle tracing in non-inertial microfluidics devices. Microsyst Technol:1-9

24. Bowman Timothy J, German D, Joelle F (2013) Inertia and scaling in deterministic lateral displacement. Biomicrofluidics 7(6):064111

25. Richard HL, Cox Edward C, Austin Robert H, Sturm James C (2004) Continuous particle separation through deterministic lateral displacement. Science 304(5673):987-990
26. Davis John A, Inglis David W, Morton Keith J, Lawrence David A, Huang Lotien R, Chou Stephen Y, Sturm James C, Austin Robert H (2006) Deterministic hydrodynamics: taking blood apart. Proc Natl Acad Sci 103(40):14779-14784

27. Zheng S, Yung R, Tai Y-C, Kasdan H (2005) Deterministic lateral displacement mems device for continuous blood cell separation. In: 18th IEEE international conference on micro electro mechanical systems, 2005. MEMS 2005, IEEE, pp 851-854

28. Okano H, Konishi T, Suzuki T, Suzuki T, Ariyasu S, Aoki S, Abe R, Hayase M (2015) Enrichment of circulating tumor cells in tumorbearing mouse blood by a deterministic lateral displacement microfluidic device. Biomed Microdevices 17(3):59

29. Mehendale N, Sharma O, D'Costa C, Paul D (2018) A radial pillar device (rapid) for continuous and high-throughput separation of multi-sized particles. Biomed Microdevices 20(1):6

30. Inglis DW, Morton KJ, Davis JA, Zieziulewicz TJ, Lawrence DA, Austin RH, Sturm JC (2008) Microfluidic device for label-free measurement of platelet activation. Lab on a Chip 8(6):925-931

31. Loutherback K, D'Silva J, Liu L, Wu A, Austin RH, Sturm JC (2012) Deterministic separation of cancer cells from blood at $10 \mathrm{ml} / \mathrm{min}$. AIP Adv 2(4):042107

32. Au SH, Edd J, Stoddard AE, Wong KHK, Fachin F, Maheswaran S, Haber DA, Stott SL, Kapur R, Toner M (2017) Microfluidic isolation of circulating tumor cell clusters by size and asymmetry. Sci Rep 7(1):2433

33. Nora Dickson $M$, Tsinberg $P$, Tang Z, Bischoff FZ, Wilson T, Leonard EF (2011) Efficient capture of circulating tumor cells with a novel immunocytochemical microfluidic device. Biomicrofluidics 5(3):034119

34. Inglis DW, Herman N, Vesey G (2010) Highly accurate deterministic lateral displacement device and its application to purification of fungal spores. Biomicrofluidics 4(2):024109

35. Beech JP, Ho BD, Garriss G, Oliveira V, Henriques-Normark B, Tegenfeldt JO (2018) Separation of pathogenic bacteria by chain length. Anal Chim Acta 1000:223-231

36. Holm SH, Beech JP, Barrett MP, Tegenfeldt JO (2011) Separation of parasites from human blood using deterministic lateral displacement. Lab on a Chip 11(7):1326-1332

37. Wunsch BH, Smith JT, Gifford SM, Wang C, Brink M, Bruce RL, Austin $\mathrm{RH}$, Stolovitzky G, Astier Y (2016) Nanoscale lateral displacement arrays for the separation of exosomes and colloids down to $20 \mathrm{~nm}$. Nat Nanotechnol 11(11):936

38. Zeming KK, Salafi T, Shikha S, Zhang Y (2018) Fluorescent labelfree quantitative detection of nano-sized bioparticles using a pillar array. Nat Commun 9(1):1254

39. Inglis DW, Davis JA, Austin RH, Sturm JC (2006) Critical particle size for fractionation by deterministic lateral displacement. Lab on a Chip 6(5):655-658

40. Inglis DW (2009) Efficient microfluidic particle separation arrays. Appl Phys Lett 94(1):013510

41. Feng S, Skelley AM, Anwer AG, Liu G, Inglis DW (2017) Maximizing particle concentration in deterministic lateral displacement arrays. Biomicrofluidics 11(2):024121

42. Inglis D (2007) Microfluidic devices for cell separation. Princeton University, Princeton

43. Bruus $\mathrm{H}$ (2008) Theoretical microfluidics, vol 18. Oxford University Press, Oxford

Publisher's Note Springer Nature remains neutral with regard to jurisdictional claims in published maps and institutional affiliations. 